\title{
Rectal versus intramuscular diclofenac in prevention of post-endoscopic retrograde cholangiopancreatography pancreatitis: experience of a Greek tertiary referral center
}

\author{
Ioannis Kalantzis ${ }^{\mathrm{a}}$, Androniki Poulou ${ }^{\mathrm{a}}$, Athanasios Papatheodorou ${ }^{\mathrm{b}}$, Konstantinos Gkoumas ${ }^{\mathrm{a}}$ \\ Korgialeneio-Mpenakeio Hellenic Red Cross Hospital, Athens, Greece
}

Abstract

Background Independent patient-related and procedure-related factors increase the risk of
pancreatitis after endoscopic retrograde cholangiopancreatography (post-ERCP pancreatitis
[PEP]). Non-steroidal anti-inflammatory drugs (NSAIDs) have demonstrated efficacy in reducing
the incidence of PEP. This study investigated the difference in the incidence of PEP between
intramuscular and rectal prophylactic administration of diclofenac before ERCP.

Methods We performed a retrospective analysis of data from 516 patients who underwent ERCP during the period 2014-2017. The route of diclofenac administration (rectal or intramuscular), patient-related and procedure-related risk factors, as well as serum amylase levels $18 \mathrm{~h}$ after the endoscopic procedure and immediate bleeding during ERCP were recorded and evaluated.

Results The overall incidence of PEP was $4.5 \%$, without significant differences between the rectal (5.2\%) and intramuscular (3.9\%) routes of administration. The factor that appeared to be of significance was pre-cut sphincterotomy, since patients who underwent that procedure showed a higher probability of PEP ( $\mathrm{P}=0.05$; odds ratio $2.67,95 \%$ confidence interval). Intraprocedural bleeding was almost twice as frequent in the rectal compared to the intramuscular group. Pancreatic stent placement did not appear to be statistically significant in the prevention of PEP, either alone or in combination with diclofenac administration.

Conclusions The results of our study did not reveal any statistically significant difference between the rectal or intramuscular administration of diclofenac in the prevention of PEP, contradicting the results of the majority of studies and meta-analyses published so far. One of the known risk factors associated with increased risk of PEP was also confirmed.

Keywords endoscopic retrograde cholangiopancreatography, post-ERCP pancreatitis, nonsteroidal anti-inflammatory drugs

Ann Gastroenterol 2020; 33 (4): 1-6

\section{Introduction}

Pancreatitis is the most common adverse event after endoscopic retrograde cholangiopancreatography (ERCP), with

Department of a Gastroenterology (Ioannis Kalantzis, Androniki Poulou, Konstantinos Gkoumas); 'Radiology (Athanasios Papatheodorou), Korgialeneio-Mpenakeio Hellenic Red Cross Hospital, Athens, Greece

Conflict of Interest: None

Correspondence to: Ioannis Kalantzis, MD, Department of Gastroenterology, Korgialeneio-Mpenakeio Hellenic Red Cross Hospital, 2 Athanasaki Street, Athens, Greece, e-mail: johnkalantzis@ hotmail.com

Received 5 August 2019; accepted 15 November 2019; published online 10 May 2020

DOI: https://doi.org/10.20524/aog.2020.0487 an incidence of $3.5 \%$ in unselected patients [1]. Various patientrelated and procedure-related risk factors have been implicated in post-ERCP pancreatitis (PEP) [1-3]. Among the drugs that have been used to prevent PEP, only non-steroidal antiinflammatory drugs (NSAIDs: diclofenac or indomethacin) have so far demonstrated their efficiency by reducing the percentage and severity of PEP in both low- and high-risk patients $[1,4-7]$. The majority of previously published studies and meta-analyses support the superiority of per rectum (PR) vs. intramuscular (IM) administration of NSAIDs in the prevention of PEP [8]. Recognition of the risk factors in each patient, as well as administration of NSAIDs before or immediately after ERCP, can lead to higher success rates for the endoscopic process and lower rates of adverse events. The purpose of this study was to investigate differences in the incidence of PEP, as well as the incidence of intraprocedural hemorrhage, in patients receiving diclofenac either PR or IM prior to ERCP. A secondary aim 
was the investigation of patient-related and procedure-related risk factors for PEP manifestation, and the protective role of pancreatic stent placement.

\section{Patients and methods}

\section{Data source and study population}

The Institutional Review Board of our hospital granted permission for a retrospective collection of data from the records of patients who underwent ERCP during the 4-year period 2014-2017. Diclofenac administration (PR or IM), patientrelated (female sex, previous $\mathrm{PEP}$, age $<50$ years old, non-dilated extrahepatic bile ducts, and normal serum bilirubin) and procedure-related (duration of cannulation attempts $>10 \mathrm{~min}$, pancreatic guidewire passages $>1$, pancreatic injection, pre-cut sphincterotomy, biliary balloon sphincter dilation, and failure to clear bile duct stones) risk factors, as well as the serum amylase level $18 \mathrm{~h}$ after the endoscopic procedure and the immediate bleeding during ERCP, were recorded. The amylase value was assessed in all patients $18 \mathrm{~h}$ after the procedure (earlier or later when clinically indicated). None of the patients underwent intraductal ultrasound or pancreatic sphincterotomy. We excluded patients in whom diclofenac was contraindicated, as well as patients with missing results of serum amylase $18 \mathrm{~h}$ post-ERCP and those who had incomplete clinical information from the medical record or a lack of imaging studies confirming the occurrence of pancreatitis. PEP diagnosis was based on the Atlanta classification criteria, which require 2 of the following: a) abdominal pain compatible with acute pancreatitis; b) serum amylase at least 3 times above the normal limit; and c) findings of acute pancreatitis on abdominal computed tomography (CT) or other imaging method [9]. Patients with abdominal pain whose amylase value was not 3 times above the upper limit of normal (normal values: $28-100 \mathrm{U} / \mathrm{L}$ ) were referred for CT scanning. Thus all patients diagnosed with PEP fulfilled at least 2 of the Atlanta diagnostic criteria. All patients were classified into 2 groups according to their indication for ERCP: choledocholithiasis or benign/malignant biliary/pancreatic stenosis.

\section{ERCP procedure}

All patients signed an informed consent form prior to endoscopic intervention. They fasted for $8 \mathrm{~h}$ and received 100 mg PR or $75 \mathrm{mg}$ IM administration of diclofenac half to $1 \mathrm{~h}$ before the endoscopy. No patient received aggressive hydration after the endoscopic procedure [10,11], antibiotics or other drugs associated with the prevention of PEP [1]. Antiplatelet therapy was discontinued one week prior to ERCP, while other anticoagulants were discontinued $24-48 \mathrm{~h}$ before the endoscopic procedure, according to the administered regimen. All ERCPs were performed by an endoscopist experienced in biliary/ pancreatic diseases, with the active involvement of trainees and in the presence of specialist nursing staff. The attempts at common bile duct cannulation were performed with the assistance of a hydrophilic guidewire, while the use of pre-cut sphincterotomy was limited to cases where cannulation was not achieved after 10 min. A pancreatic stent was placed in all patients who had pancreatic duct cannulation more than once, and in $79 \%$ of patients (41/52) with pancreatic duct injection, in order to reduce the incidence of PEP. An anesthesiologist was responsible for the administration of sedation and monitoring of the patients' vital signs during the endoscopy. Patients could consume a liquid diet $6 \mathrm{~h}$ after ERCP if they did not experience abdominal pain, fever or other adverse events. Vital signs, symptoms and laboratory values were monitored for at least $24 \mathrm{~h}$ after the procedure.

\section{Statistical analysis}

Statistical analysis was performed using SPSS Version 24. Factors associated with an increased risk for PEP were examined by univariate statistics (chi-square with continuity correction and Fisher's exact tests, as appropriate) and multivariate analyses (logistic regression method). All study variables (indication, pancreatic stent insertion, NSAID administration (PR or IM), patient-related and procedure-related risk factors) were included in the multivariate analysis. The distribution of amylase between the study groups was analyzed by Student's $t$-test. Amylase values were transformed into the natural logarithm in order to decrease the variability. Reported amylase summary was based on the geometric mean (gmean) followed by the corresponding $95 \%$ confidence interval (CI). All tests were 2 -sided and statistical significance was set at $\mathrm{P}<0.05$.

\section{Results}

During the study period (2014-2017) 688 patients underwent ERCP in our department. One hundred seventy-two patients were excluded because of a lack of information in their medical records, missing values of post-ERCP amylase, or nonadministration of diclofenac (contradiction or allergy). Finally, a total of 516 patients (294 men, 222 women) of mean age 72.5 years (range 26-99) were included in the study. The indication was either choledocholithiasis $(\mathrm{N}=351,68 \%)$ or benign/ malignant biliary/pancreatic stenosis ( $\mathrm{N}=165,32 \%)$ (Fig. 1). During the 2-year period 2014-2015 all patients ( $\mathrm{N}=233,45 \%)$ received PR administration of diclofenac, whereas during 20162017 diclofenac was administered IM ( $\mathrm{N}=283,55 \%)$, because of availability issues in our pharmaceutical department. No patient received either PR or IM diclofenac based on any interventional study or protocol. Basic characteristics of each group, including PEP-related factors, are given in Table 1. Previous PEP, precut sphincterotomy and duration of cannulation attempts $>10$ min were statistically more frequent in patients who received diclofenac IM, while non-dilated extrahepatic bile ducts and pancreatic injection were more frequent in the PR group. The total incidence of PEP was $4.5 \%(\mathrm{~N}=23)$, with no statistically significant differences between the PR $(\mathrm{N}=12,5.2 \%)$ and $\mathrm{IM}(\mathrm{N}=11,3.9 \%)$ 


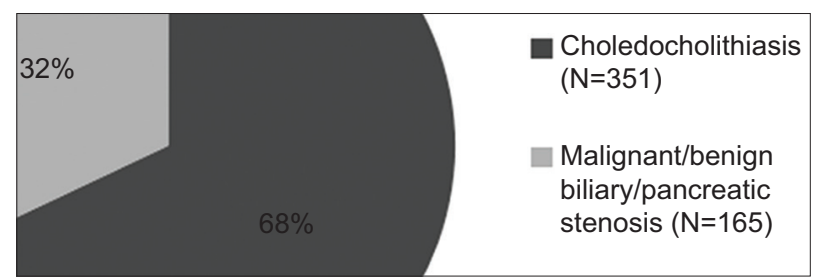

Figure 1 Indications for endoscopic retrograde cholangiopancreatography

Table 1 Basic characteristics of the patients

\begin{tabular}{|c|c|c|c|}
\hline \multirow[t]{2}{*}{ Characteristics } & $\begin{array}{l}\text { NSAID-PR } \\
\mathrm{N}=233\end{array}$ & $\begin{array}{l}\text { NSAID-IM } \\
\mathrm{N}=283\end{array}$ & \multirow[t]{2}{*}{ P-value } \\
\hline & $\mathrm{n}(\%)$ & $\mathrm{n}(\%)$ & \\
\hline \multicolumn{4}{|l|}{ Indication } \\
\hline Choledocholithiasis & $169(72.5)$ & $182(64.3)$ & 0.058 \\
\hline $\begin{array}{l}\text { Malignant/benign biliary/ } \\
\text { pancreatic stenosis }\end{array}$ & $64(27.5)$ & $101(35.7)$ & \\
\hline \multicolumn{4}{|l|}{ Patient related factors } \\
\hline Female & $102(43.8)$ & $120(42.7)$ & 0.822 \\
\hline Age $<50$ years & $15(6.5)$ & $26(9.2)$ & 0.338 \\
\hline Normal serum bilirubin & $105(45.1)$ & $130(45.9)$ & 0.913 \\
\hline History of pancreatitis & $6(2.6)$ & $5(1.8)$ & 0.744 \\
\hline Previous PEP & $0(0)$ & $14(4.9)$ & 0.002 \\
\hline $\begin{array}{l}\text { Non-dilated extrahepatic } \\
\text { bile ducts }\end{array}$ & $66(29.7)$ & $58(20.6)$ & 0.025 \\
\hline \multicolumn{4}{|l|}{ Procedure related factors } \\
\hline $\begin{array}{l}\text { Failure to clear bile duct } \\
\text { stones }\end{array}$ & $30(12.9)$ & $30(10.5)$ & 0.507 \\
\hline Pre-cut sphincterotomy & $25(10.7)$ & $65(23.0)$ & $<0.001$ \\
\hline $\begin{array}{l}\text { Cannulation attempts } \\
\text { duration }>10 \mathrm{~min}\end{array}$ & $55(23.6)$ & $94(33.2)$ & 0.021 \\
\hline $\begin{array}{l}\text { Biliary balloon sphincter } \\
\text { dilation }\end{array}$ & $61(26.2)$ & $73(25.8)$ & $>0.999$ \\
\hline Pancreatic injection & $31(13.3)$ & $21(7.4)$ & 0.039 \\
\hline $\begin{array}{l}\text { Pancreatic guidewire } \\
\text { passages }>1\end{array}$ & $13(5.6)$ & $24(8.5)$ & 0.271 \\
\hline Pancreatic stent placement & $14(6.0)$ & $27(9.5)$ & 0.189 \\
\hline
\end{tabular}

NSAID, non-steroid anti-inflammatory drug; $P R$, per rectum; IM, intramuscular; PEP, post-endoscopic retrograde cholangiopancreatography pancreatitis

routes of administration $(\mathrm{P}=0.633)$ (Fig. 2). In univariate analysis, regarding patient-related and procedure-related risk factors, the factor that appeared to be of statistical significance was pre-cut sphincterotomy, since patients who underwent that procedure had a 2.67 fold higher probability of PEP $(\mathrm{P}=0.05)$. Younger age $(<50)$, female sex, history of pancreatitis or PEP, passage of pancreatic guidewire more than once, non-dilated extrahepatic bile ducts, pancreatic injection, normal serum bilirubin, balloon biliary sphincter dilation and failure to clear bile duct stones did

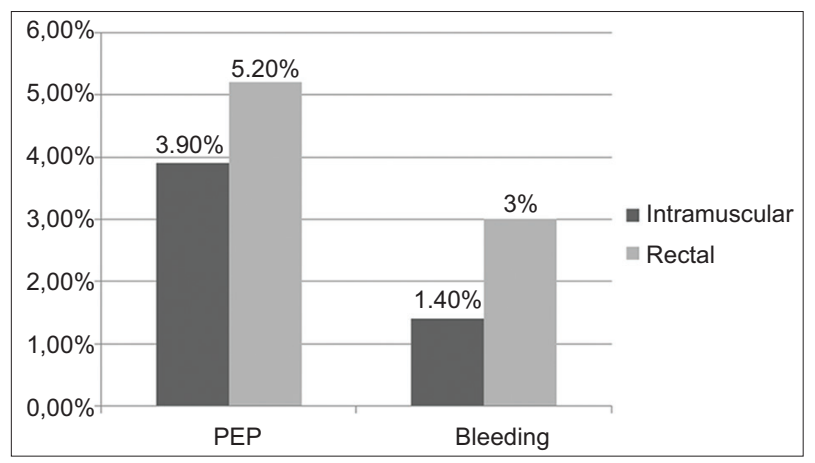

Figure 2 Association of post-endoscopic retrograde cholangiopancreatography pancreatitis (PEP) and intraprocedural bleeding with intramuscular and rectal administration of diclofenac

not show any statistical significance as risk factors for PEP (Table 2). Intraprocedural bleeding $(\mathrm{N}=11,2.1 \%)$ was almost twice as frequent in the PR $(\mathrm{N}=7,3 \%)$ as in the IM group $(\mathrm{N}=4,1.4 \%)$, but the difference did not reach statistical significance (Fig. 2). Most of the cases of intraprocedural bleeding ( 8 of 11) were treated with injection of adrenaline solution $(1: 10,000)$ while the remaining 3 were treated with the assistance of the balloon used during sphincter dilation. Pancreatic stent placement did not show any statistical significance in the prevention of PEP, either alone or in combination with diclofenac (IM or PR) administration. The gmean amylase value in PEP incidents did not show any statistically significant difference between the 2 NSAIDs subgroups (PR: 1317 U/L, 95\%CI 1020-1699 vs. IM: $1179 \mathrm{U} / \mathrm{L}, 95 \% \mathrm{CI} 741-1874 ; \mathrm{P}=0.639)$, nor in patients who had hyperamylasemia after ERCP (PR: 230 U/L, 95\%CI 198-268 vs. IM: $202 \mathrm{U} / \mathrm{L}, 95 \% \mathrm{CI}$ 179-227; $\mathrm{P}=0.172$ ), although higher gmean values were noted in the PR subgroup. In the multivariate analysis, all study variables (patient-related and procedure-related factors, indication, pancreatic stent insertion and NSAID administration) were included. Only pre-cut sphincterotomy was confirmed as a significant and independent factor for PEP ( $\mathrm{P}=0.029)$, with these patients displaying 2.7 times greater probability of PEP manifestation (Table 2). Interestingly, between the various combinations of the above mentioned risk factors, patients who had both pre-cut sphincterotomy and failure to clear bile duct stones presented the highest incidence of PEP $(30 \%, \mathrm{P}=0.001)$.

\section{Discussion}

ERCP remains an indispensable therapeutic endoscopic procedure in the treatment of biliary and pancreatic diseases. Acute pancreatitis is one of the most common adverse events, with rates ranging from $3.5-30 \%$ in high-risk patients $[1,8,11,12]$. Evaluation of the patient-related and procedure-related factors associated with the occurrence of PEP, as well as the application of preventive measures (NSAIDs, pancreatic stent placement), have been widely adopted with a view to reducing the incidence of this adverse event $[1,2,13]$. Several pharmaceutical agents, including octreotide, somatostatin, protease inhibitors, interleukin-10, and glyceryl nitrate, have been tested for the 
4 I. Kalantzis et al

Table 2 Association of study variables with PEP

\begin{tabular}{|c|c|c|c|c|c|c|c|c|}
\hline \multirow[t]{2}{*}{ Variable } & \multicolumn{2}{|c|}{ Presence of PEP N=23 } & \multicolumn{2}{|c|}{ Absence of PEP $\mathrm{N}=493$} & \multicolumn{2}{|c|}{ Univariate analysis } & \multicolumn{2}{|c|}{ Logistic regression } \\
\hline & $\mathrm{n}$ & $\%$ & $\mathrm{n}$ & $\%$ & $\mathrm{P}$ & OR, 95\%CI & $\mathrm{P}$ & OR, $95 \% \mathrm{CI}$ \\
\hline Sex & & & & & 0.262 & & 0.179 & \\
\hline Male & 10 & 3.4 & 284 & 96.6 & & & & \\
\hline Female & 13 & 5.9 & 209 & 94.1 & & & & \\
\hline Age & & & & & 0.243 & & 0.134 & \\
\hline$<50$ years & 0 & 0.0 & 41 & 100.0 & & & & \\
\hline$>50$ years & 23 & 4.9 & 450 & 95.1 & & & & \\
\hline Indication & & & & & 0.600 & & 0.633 & \\
\hline $\begin{array}{l}\text { Benign-malignant Biliary/ } \\
\text { Pancreatic stenosis/ }\end{array}$ & 9 & 5.5 & 156 & 94.5 & & & & \\
\hline Choledocholithiasis & 14 & 4.0 & 337 & 96.0 & & & & \\
\hline Pancreatic injection & & & & & 0.719 & & 0.174 & \\
\hline Yes & 22 & 4.7 & 442 & 95.3 & & & & \\
\hline No & 1 & 1.9 & 51 & 98.1 & & & & \\
\hline NSAID & & & & & 0.633 & & 0.259 & \\
\hline NSAID-PR & 12 & 5.2 & 221 & 94.8 & & & & \\
\hline NSAID-IM & 11 & 3.9 & 272 & 96.1 & & & & \\
\hline Failure to clear bile duct stones & & & & & 0.328 & & 0.467 & \\
\hline Yes & 4 & 6.7 & 56 & 93.3 & & & & \\
\hline No & 19 & 4.2 & 437 & 95.8 & & & & \\
\hline Normal serum bilirubin & & & & & 0.398 & & 0.359 & \\
\hline Increased & 15 & 5.3 & 266 & 94.7 & & & & \\
\hline Normal & 8 & 3.4 & 227 & 96.6 & & & & \\
\hline Non-dilated extrahepatic bile ducts & & & & & 0.365 & & $\mathrm{NA}^{1}$ & \\
\hline Yes & 8 & 6.5 & 116 & 93.5 & & & & \\
\hline No & 15 & 4.0 & 364 & 96.0 & & & & \\
\hline History of pancreatitis & & & & & 0.397 & & 0.658 & \\
\hline Yes & 1 & 9.1 & 10 & 90.9 & & & & \\
\hline No & 22 & 4.4 & 483 & 95.6 & & & & \\
\hline Previous PEP & & & & & 1 & & 0.467 & \\
\hline Yes & 0 & 0.0 & 14 & 100.0 & & & & \\
\hline No & 23 & 4.6 & 479 & 95.4 & & & & \\
\hline Pre-cut sphincterotomy & & & & & 0.050 & $\begin{array}{c}2.67 \\
{[1.10-6.51]}\end{array}$ & 0.029 & $\begin{array}{c}2.70 \\
{[1.10-6.58]}\end{array}$ \\
\hline Yes & 8 & 8.9 & 82 & 91.1 & & & & \\
\hline No & 15 & 3.5 & 411 & 96.5 & & & & \\
\hline $\begin{array}{l}\text { Cannulation attempts duration } \\
>10 \text { min }\end{array}$ & & & & & 0.069 & & 0.485 & \\
\hline Yes & 11 & 7.4 & 138 & 92.6 & & & & \\
\hline No & 12 & 3.3 & 355 & 96.7 & & & & \\
\hline
\end{tabular}


Table 2 (Continued)

\begin{tabular}{|c|c|c|c|c|c|c|c|c|}
\hline \multirow[t]{2}{*}{ Variable } & \multicolumn{2}{|c|}{ Presence of PEP N=23 } & \multicolumn{2}{|c|}{ Absence of PEP $\mathrm{N}=493$} & \multicolumn{2}{|c|}{ Univariate analysis } & \multicolumn{2}{|c|}{ Logistic regression } \\
\hline & $\mathrm{n}$ & $\%$ & $\mathrm{n}$ & $\%$ & $\mathrm{P}$ & OR, 95\%CI & $\mathrm{P}$ & OR, $95 \% \mathrm{CI}$ \\
\hline Biliary balloon sphincter dilation & & & & & 0.818 & & 0.953 & \\
\hline Yes & 5 & 3.7 & 129 & 96.3 & & & & \\
\hline No & 18 & 4.7 & 364 & 95.3 & & & & \\
\hline Pancreatic guidewire passages $>1$ & & & & & 0.074 & & 0.272 & \\
\hline Yes & 4 & 10.8 & 33 & 89.2 & & & & \\
\hline No & 19 & 4.0 & 460 & 96.0 & & & & \\
\hline Pancreatic stent insertion & & & & & 0.703 & & 0.540 & \\
\hline Yes & 2 & 4.9 & 39 & 95.1 & & & & \\
\hline No & 21 & 4.4 & 454 & 95.6 & & & & \\
\hline
\end{tabular}

${ }^{1} \mathrm{NA}=$ Non-available: The variable "non-dilated extrahepatic bile ducts" was not included in the logistic regression because of the 13 missing values, in order to avoid reducing the number of patients entering the model

ERCP, endoscopic retrograde cholangiopancreatography; PEP, post-ERCP pancreatitis; NSAID, non-steroid anti-inflammatory drug; PR, per rectum; IM, intramuscular; $\mathrm{OR}$, odds ratio; $\mathrm{CI}$, confidence interval

prevention of PEP without satisfactory results, but NSAIDs (diclofenac or indomethacin) have shown their efficacy in reducing the incidence of PEP $[1,8,11,12]$.

In our study, the primary endpoint was any statistically significant difference between the PR and IM administration of diclofenac as regards the incidence of PEP. The metaanalyses published so far support the efficacy of PR over IM administration of diclofenac and demonstrate the efficacy of NSAIDs in the prevention of moderate to severe PEP in both average and high-risk patients $[8,14,15]$. However, one study demonstrated the efficacy of IM administration of diclofenac [11], while another showed no difference between PR and IM administration [16]. The present study found no statistically significant differences between PR and IM administration of NSAIDs in moderate or severe pancreatitis, or in patients with moderate or high risk. An earlier metaanalysis supported the efficacy of NSAIDs prior to ERCP [17], while more recent meta-analyses did not reveal any difference between pre- and post-ERCP administration [8,15]. All patients of our study received diclofenac before the endoscopy and we found no difference in PEP occurrence rates between the 2 subgroups (PR vs. IM).

Pancreatic stent placement contributes to the prevention of PEP [1-4,11]. In one randomized controlled trial, indomethacin was administered in addition to pancreatic stent placement in high-risk patients [18], while a meta-analysis showed that the combination of PR NSAIDs and stents is not superior to either approach alone [19]. In our study, the combination of pancreatic stent placement and diclofenac administration, either IM or PR, did not appear to lead to a further reduction in the incidence of PEP.

The rate of intraprocedural bleeding in our study was in accordance with the existing literature [2,20]. Although the rate of intraprocedural hemorrhage was twice as high in the PR compared to the IM group, this difference was not statistically significant. Differences in mean amylase values in
PEP patients, as well as in those who experienced post-ERCP hyperamylasemia, between the PR and IM administration of diclofenac have not been widely investigated [16]. In our study, the mean amylase value in patients who developed PEP did not show any statistically significant difference between the 2 NSAIDs groups, nor did it differ when the analysis was limited to patients who had hyperamylasemia after ERCP.

Patient-related and procedure-related factors related to the appearance of PEP were included in the multivariate analysis. Only pre-cut sphincterotomy showed a statistically significant relation, in accordance with the existing literature [1-3,13]. The absence of statistical significance for the other risk factors can be attributed to the relatively small number of patients in our study. This parameter, along with the retrospective nature of the investigation, may represent limitations of the study.

In conclusion, administration of NSAIDs before or immediately after ERCP is supported by several meta-analyses and is a cheap, easy to administer and effective measure without major adverse events or contraindications. Numerous studies support the superiority of PR compared to IM administration of diclofenac in reducing PEP. PR administration of diclofenac is easier and less painful compared to IM administration, while there is no significant difference between them regarding cost. Although diclofenac should be administered PR in all patients, according to the results of this study, in certain cases (absence of diclofenac suppositories or patient's refusal of PR administration) the IM route could be considered.

In our study, the overall incidence of PEP was in line with previous studies, with no differences between the 2 different routes of administration (PR or IM), a result that needs further elucidation. Assessing the absolute and relative indications for therapeutic endoscopy and avoiding diagnostic ERCPs should be the first step before performing an invasive endoscopic procedure. Careful evaluation of the characteristics of patients undergoing ERCP (age, sex, history of pancreatitis or PEP, sphincter of Oddi dysfunction), as well as laboratory 
measurements (serum bilirubin) and imaging (extrahepatic bile duct diameter), are essential in reducing the rate of PEP. Implementation of specific measures during the endoscopy (pancreatic stent placement, early pre-cut sphincterotomy, guidewire-assisted cannulation) further contribute in this direction. In cases of adverse events, early recognition and prompt intervention are essential to minimize prolonged hospitalization, late complications, morbidity and mortality.

\section{Summary Box}

\section{What is already known:}

- Endoscopic retrograde cholangiopancreatography (ERCP) remains an essential endoscopic procedure in the treatment of pancreatobiliary diseases

- Post-ERCP pancreatitis (PEP) is the most common and possibly serious adverse event of ERCP

- Various patient-related and procedure-related risk factors have been implicated in PEP

- Non-steroidal anti-inflammatory drugs have demonstrated their efficacy in reducing the prevalence and severity of PEP in both low- and high-risk patients

\section{What the new findings are:}

- No statistically significant differences between the rectal and intramuscular administration of diclofenac before ERCP were observed in the incidence of PEP

- Pre-cut sphincterotomy was identified as the only procedure-related factor for PEP incidence

- Intraprocedural bleeding and mean amylase values showed no statistically significant difference between the different routes of diclofenac administration

\section{References}

1. Dumonceau JM, Andriulli A, Elmunzer BJ, et al; European Society of Gastrointestinal Endoscopy. Prophylaxis of post-ERCP pancreatitis: European Society of Gastrointestinal Endoscopy (ESGE) Guideline - updated June 2014. Endoscopy 2014;46:799-815.

2. Cotton PB, Garrow DA, Gallagher J, Romagnuolo J. Risk factors for complications after ERCP: a multivariate analysis of 11,497 procedures over 12 years. Gastrointest Endosc 2009;70:80-88.

3. Testoni PA, Mariani A, Aabakken L, et al. Papillary cannulation and sphincterotomy techniques at ERCP: European Society of
Gastrointestinal Endoscopy (ESGE) Clinical Guideline. Endoscopy 2016;48:657-683.

4. Wong LL, Tsai HH. Prevention of post-ERCP pancreatitis. World J Gastrointest Pathophysiol 2014;5:1-10.

5. Elmunzer BJ, Waljee AK, Elta GH, Taylor JR, Fehmi SM, Higgins PD. A meta-analysis of rectal NSAIDs in the prevention of post-ERCP pancreatitis. Gut 2008;57:1262-1267.

6. Dai HF, Wang XW, Zhao K. Role of nonsteroidal anti-inflammatory drugs in the prevention of post-ERCP pancreatitis: a meta-analysis. Hepatobiliary Pancreat Dis Int 2009;8:11-16.

7. Ding X, Chen M, Huang S, Zhang S, Zou X. Nonsteroidal antiinflammatory drugs for prevention of post-ERCP pancreatitis: a meta-analysis. Gastrointest Endosc 2012;76:1152-1159.

8. Patai Á, Solymosi N, Mohácsi L, Patai ÁV. Indomethacin and diclofenac in the prevention of post-ERCP pancreatitis: a systematic review and meta-analysis of prospective controlled trials. Gastrointest Endosc 2017;85:1144-1156.

9. Banks PA, Bollen TL, Dervenis C, et al; Acute Pancreatitis Classification Working Group. Classification of acute pancreatitis-2012: revision of the Atlanta classification and definitions by international consensus. Gut 2013;62:102-111.

10. Wu D, Wan J, Xia L, Chen J, Zhu Y, Lu N. The efficiency of aggressive hydration with lactated ringer solution for the prevention of postERCP pancreatitis: A Systematic review and meta-analysis. J Clin Gastroenterol 2017;51:e68-e76.

11. Senol A, Saritas U, Demirkan H. Efficacy of intramuscular diclofenac and fluid replacement in prevention of post-ERCP pancreatitis. World J Gastroenterol 2009;15:3999-4004.

12. Zhao XW, Bao JJ, Hu C, et al. Effect of diclofenac on the levels of lipoxin A4 and resolvin D1 and E1 in the post-ERCP pancreatitis. Dig Dis Sci 2014;59:2992-2996.

13. Williams EJ, Taylor S, Fairclough P, et al. Risk factors for complication following ERCP; results of a large-scale, prospective multicenter study. Endoscopy 2007;39:793-801.

14. Li X, Tao LP, Wang CH. Effectiveness of nonsteroidal antiinflammatory drugs in prevention of post-ERCP pancreatitis: a meta-analysis. World J Gastroenterol 2014;20:12322-12329.

15. Puig I, Calvet X, Baylina M, et al. How and when should NSAIDs be used for preventing post-ERCP pancreatitis? A systematic review and meta-analysis. PLoS One 2014;9:e92922.

16. Uçar R, Biyik M, Uçar E, et al. Rectal or intramuscular diclofenac reduces the incidence of pancreatitis after endoscopic retrograde cholangiopancreatography. Turk J Med Sci 2016;46:1059-1063.

17. Rustagi T, Njei B. Factors affecting the efficacy of nonsteroidal antiinflammatory drugs in preventing post-endoscopic retrograde cholangiopancreatography pancreatitis: a systematic review and meta-analysis. Pancreas 2015;44:859-867.

18. Elmunzer BJ, Scheiman JM, Lehman GA, et al; U.S. Cooperative for Outcomes Research in Endoscopy (USCORE). A randomized trial of rectal indomethacin to prevent post-ERCP pancreatitis. $N$ Engl J Med 2012;366:1414-1422.

19. Akbar A, Abu Dayyeh BK, Baron TH, Wang Z, Altayar O, Murad MH. Rectal nonsteroidal anti-inflammatory drugs are superior to pancreatic duct stents in preventing pancreatitis after endoscopic retrograde cholangiopancreatography: a network meta-analysis. Clin Gastroenterol Hepatol 2013;11:778-783.

20. Anderson MA, Fisher L, Jain R, et al; ASGE Standards of Practice Committee. Complications of ERCP. Gastrointest Endosc 2012;75:467-473. 